\title{
MINIMAL VARIANCE HEDGING FOR FRACTIONAL BROWNIAN MOTION *
}

\author{
FRANCESCA BIAGINI ${ }^{\dagger}$ AND BERNT $\varnothing K S E N D A L \ddagger$
}

\begin{abstract}
We discuss the extension to the multi-dimensional case of the Wick-Itô integral with respect to fractional Brownian motion, introduced by [6] in the 1-dimensional case. We prove a multidimensional Itô type isometry for such integrals, which is used in the proof of the multi-dimensional Itô formula. The results are applied to study the problem of minimal variance hedging in a market driven by fractional Brownian motions.
\end{abstract}

1. Introduction. In the following we let $H=\left(H_{1}, H_{2}, \ldots, H_{m}\right)$ be an $m$ dimensional Hurst vector with components $H_{i} \in\left(\frac{1}{2}, 1\right)$ for $i=1,2, \ldots, m$, and we let $B^{(H)}(t)=\left(B_{1}^{(H)}(t), \ldots, B_{m}^{(H)}(t)\right)$ be an $m$-dimensional fractional Brownian motion $(f B m)$ with Hurst parameter $H$. This means that $B^{(H)}(t)=B^{(H)}(t, \omega) ; t \in \mathbb{R}$, $\omega \in \Omega$ is a continuous Gaussian stochastic process on a filtered probability space $\left(\Omega, \mathcal{F}_{t}^{(H)}, \mu\right)$ with mean

$$
\mathbb{E}\left[B^{(H)}(t)\right]=0=B^{(H)}(0) \quad \text { for all } t
$$

and covariance

$$
\mathbb{E}\left[B_{i}^{(H)}(s) B_{j}^{(H)}(t)\right]=\frac{1}{2}\left\{|s|^{2 H_{i}}+|t|^{2 H_{i}}-|s-t|^{2 H_{i}}\right\} \delta_{i j}
$$

where

$$
\delta_{i j}= \begin{cases}0 & \text { if } \quad i \neq j \\ 1 & \text { if } \quad i=j ; \quad i \leq i, j \leq m\end{cases}
$$

where $\mathbb{E}=\mathbb{E}_{\mu}$ denotes the expectation with respect to the probability law $\mu$ of $B^{(H)}(\cdot)$.

In other words, $B^{(H)}(t)$ consists of $m$ independent 1-dimensional fractional Brownian motions with Hurst parameters $H_{1}, \ldots, H_{m}$, respectively. If $H_{i}=\frac{1}{2}$ for all $i$, then $B^{(H)}(t)$ coincides with classical Brownian motion $B(t)$. We refer to [11], [13] and [18] for more information about 1-dimensional $f B m$. Because of its properties (persistence/antipersistence and self-similarity) $\mathrm{fBm}$ has been suggested as a useful mathematical tool in many applications, including finance [10]. For example, these features of $f \mathrm{Bm}$ seem to appear in the log-returns of stocks [18], in weather derivative models [3] and in electricity prices in a liberated electricity market [20].

In view of this it is of interest to develop a powerful calculus for $f B m$. Unfortunately, $f B m$ is not a semimartingale nor a Markov process (unless $H_{i}=\frac{1}{2}$ for all $i$ ), so these theories cannot be applied to $f B m$. However, if $H_{i}>\frac{1}{2}$ then the paths have zero quadratic variation and it is therefore possible to define a pathwise integral, denoted by

$$
\int_{\mathbb{R}} f(t, \omega) \delta B^{(H)}(t)
$$

\footnotetext{
${ }^{*}$ Received January 28, 2003; accepted for publication August 13, 2003.

${ }^{\dagger}$ Department of Mathematics, University of Bologna, Piazza di Porta S. Donato, 5, I-40127 Bologna, Italy (biagini@dm.unibo.it).

${ }_{\ddagger}^{\ddagger}$ Department of Mathematics, University of Oslo, Box 1053 Blindern, N-0316 Oslo, Norway (oksendal@math.uio.no); Norwegian School of Economics and Business Administration, Helleveien 30, N-5045 Bergen, Norway.
} 
by a classical result of Young from 1936. See [12] and the references therein. This integral will obey Stratonovich type (i.e. "deterministic") integration rules. Typically the expectation of such integrals is not 0 and it is known ([12], [15], [16], [19]) that the use of these integrals in finance will give markets with arbitrage, even in the most basic cases. In fact, this unpleasant situation (from a modelling point of view) occurs whenever we use an integration theory with Stratonovich integration rules in the generation of wealth from a portfolio. See e.g. the simple examples of [4] and [19].

Because of this - and for several other reasons - it is natural to try other types of integration with respect to $f B m$. Let $\mathcal{L}_{\phi}^{1,2}$ be the set of (measurable) processes $f(\cdot, \cdot): \mathbb{R} \times \Omega \rightarrow \mathbb{R}$ such that $\|f\|_{\mathcal{L}_{\phi}^{1,2}}<\infty$, where

$$
\|f\|_{\mathcal{L}_{\phi}^{1,2}}^{2}:=\mathbb{E}\left[\int_{\mathbb{R}} \int_{\mathbb{R}} f(s) f(t) \phi(s, t) d s d t+\left(\int_{\mathbb{R}} D_{t}^{\phi} f(t) d t\right)^{2}\right] .
$$

In [6] a Wick-Itô type of integral is constructed, denoted by

$$
\int_{\mathbb{R}} f(t, \omega) d B^{(H)}(t)
$$

where $B^{(H)}(t)$ is a 1 -dimensional $f B m$ with $H \in\left(\frac{1}{2}, 1\right)$. This integral exists as an element of $L^{2}(\mu)$ for all (measurable) processes $f(t, \omega)$ such that $\|f\|_{\mathcal{L}_{\phi}^{1,2}}<\infty$. Here, and in the following,

$$
\phi(s, t)=\phi_{H}(s, t)=H(2 H-1)|s-t|^{2 H-2} ; \quad(s, t) \in \mathbb{R}^{2}, \quad \frac{1}{2}<H<1
$$

and

$$
D_{t}^{\phi} F=\int_{\mathbb{R}} \phi(s, t) D_{s} F d s
$$

denotes the Malliavin $\phi$-derivative of $F$ (see [6, Definition 3.4]). If $f(t, \omega)$ is a step process of the form

$$
f(t, \omega)=\sum_{i=1}^{n} f_{i}(\omega) \mathcal{X}_{\left[t_{i}, t_{i+1}\right)}(t), \quad \text { where } t_{1}<t_{2}<\cdots<t_{n+1},
$$

and $\|f\|_{\mathcal{L}_{\phi}^{1,2}}<\infty$, then the integral is defined by

$$
\int_{\mathbb{R}} f(t, \omega) d B^{(H)}(t)=\sum_{i=1}^{n} f_{i}(\omega) \diamond\left(B^{(H)}\left(t_{i+1}\right)-B^{(H)}\left(t_{i}\right)\right),
$$

where $\diamond$ denotes the Wick product. We have the following basic properties of the Wick-Itô integral:

$$
\begin{aligned}
& \mathbb{E}\left[\int_{\mathbb{R}} f(t, \omega) d B^{(H)}(t)\right]=0 \quad \text { for all } f \in \mathcal{L}_{\phi}^{1,2} \\
& \mathbb{E}\left[\left(\int_{\mathbb{R}} f(t, \omega) d B^{(H)}(t)\right)\left(\int_{\mathbb{R}} g(t, \omega) d B^{(H)}(t)\right)\right]=(f, g)_{\mathcal{L}_{\phi}^{1,2}} \quad \text { for all } f, g \in \mathcal{L}_{\phi}^{1,2} \text { where } \\
& (f, g)_{\mathcal{L}_{\phi}^{1,2}}=\mathbb{E}\left[\int_{\mathbb{R}} \int_{\mathbb{R}} f(s) g(t) \phi(s, t) d s d t+\left(\int_{\mathbb{R}} D_{t}^{\phi} f(t) d t\right) \cdot\left(\int_{\mathbb{R}} D_{t}^{\phi} g(t) d t\right)\right] .
\end{aligned}
$$


See [6] for details and proofs.

This Wick-Itô fractional calculus was subsequently extended to a white noise setting and applied to finance in [9]. Later this white noise theory was generalized to all $H \in(0,1)$ by [7].

All the above papers [6], [9] and [7] only deal with the 1-dimensional case. In Section 2 of this paper we discuss the extension of this integral to the $m$-dimensional case, i.e. we discuss the integral

$$
\int_{\mathbb{R}} f(t, \omega) d B^{(H)}(t)=\sum_{i=1}^{m} \int_{\mathbb{R}} f_{i}(t, \omega) d B_{i}^{(H)}(t) \quad \text { for } f=\left(f_{1}, \ldots, f_{m}\right) \in \mathcal{L}_{\phi}^{1,2}(m)
$$

where $B^{(H)}(t)=\left(B_{1}^{(H)}(t), \ldots, B_{m}^{(H)}(t)\right)$ is $m$-dimensional $f B m, \phi=\left(\phi_{H_{1}}, \ldots, \phi_{H_{m}}\right)$ and $\mathcal{L}_{\phi}^{1,2}(m)$ is the corresponding class of integrands (see (2.5) below). We prove the $m$-dimensional analogue of the isometry (1.9), which turns out to have some unexpected features (see Theorem 2.1). By combining the multi-dimensional fractional Itô formula (Theorem 2.6) with Theorem 2.1 we obtain another fractional Itô isometry (Theorem 2.7). Finally, we end Section 2 by proving a fractional integration by parts formula (Theorem 2.9 and Theorem 2.10).

In Section 3 we apply the above results to study the problem of minimal variance hedging in a (possibly incomplete) market driven by $m$-dimensional $f B m$. Here we use fractional mathematical market model introduced by [9] and by [7]. For classical Brownian motions (and semimartingales) this problem has been studied by many researchers. See for example the survey [17] and the references therein. It turns out that for $f B m$ this problem is even harder than in the classical case and in this paper we concentrate on a special case in order to get more specific results.

2. Multi-dimensional Wick-Itô integration with respect to $f B m$. Let $B^{(H)}(t)=\left(B_{1}^{(H)}(t), \ldots, B_{m}^{(H)}(t)\right) ; t \in \mathbb{R}, \omega \in \Omega$ be $m$-dimensional $f B m$ with Hurst vector $H=\left(H_{1}, \ldots, H_{m}\right) \in\left(\frac{1}{2}, 1\right)^{m}$, as in Section 1 . Since the $B_{k}^{(H)}(\cdot)$ are independent, we may regard $\Omega$ as a product $\Omega=\Omega_{1} \times \Omega_{2} \times \cdots \times \Omega_{m}$ of identical copies $\Omega_{k}$ of some $\bar{\Omega}$ and write $\omega=\left(\omega_{1}, \ldots, \omega_{m}\right) \in \Omega$.

Let $\mathcal{F}=\mathcal{F}_{\infty}^{(m, H)}$ be the $\sigma$-algebra generated by $\left\{B_{k}^{(H)}(s, \cdot) ; s \in \mathbb{R}, k=1,2, \ldots, m\right\}$ and let $\mathcal{F}_{t}=\mathcal{F}_{t}^{(m, H)}$ be the $\sigma$-algebra generated by $\left\{B_{k}^{(H)}(s, \cdot) ; 0 \leq s \leq t, k=\right.$ $1,2, \ldots, m\}$. If $F: \Omega \rightarrow \mathbb{R}$ is $\mathcal{F}$-measurable, $1 \leq k \leq m$, we set

$$
D_{k, t}^{\phi} F=\int_{\mathbb{R}} \phi_{k}(s, t) D_{k, t} F d t \quad \text { (if the integral converges) }
$$

where

$$
\begin{gathered}
\phi=\left(\phi_{1}, \ldots, \phi_{m}\right) \\
\phi_{k}(s, t)=\phi_{H_{k}}(s, t)=H_{k}\left(2 H_{k}-1\right)|s-t|^{2 H_{k}-2} ; \quad(s, t) \in \mathbb{R}^{3}, \quad k=1,2, \ldots, m
\end{gathered}
$$

and $D_{k, t} F=\frac{\partial F}{\partial \omega_{k}}(t, \omega)$ is the Malliavin derivative of $F$ with respect to $\omega_{k}$, at $(t, \omega)$ (if it exists).

Let $\mathcal{B}=\mathcal{B}(\mathbb{R})$ denote the Borel $\sigma$-algebra on $\mathbb{R}$. Similarly to the 1-dimensional case we can define the multi-dimensional fractional Wick-Itô integral

$$
\int_{\mathbb{R}} f(t, \omega) d B^{(H)}(t)=\sum_{k=1}^{m} \int_{\mathbb{R}} f_{k}(t, \omega) d B_{k}^{(H)}(t) \in L^{2}(\mu)
$$


for all $\mathcal{B} \times \mathcal{F}$-measurable processes $f(t, \omega)=\left(f_{1}(t, \omega), \ldots, f_{m}(t, \omega)\right) \in \mathbb{R}^{m}$ such that

$$
\begin{aligned}
& \left\|f_{k}\right\|_{\mathcal{L}_{\phi_{k}}^{1,2}}<\infty \quad \text { for all } k=1,2, \ldots, m \text {, where } \\
& \left\|f_{k}\right\|_{\mathcal{L}_{\phi_{k}}^{1,2}}:=\mathbb{E}\left[\int_{\mathbb{R}} \int_{\mathbb{R}} f_{k}(s) f_{k}(t) \phi_{k}(s, t) d s d t+\left(\int_{\mathbb{R}} D_{k, t}^{\phi} f_{k}(t) d t\right)^{2}\right] .
\end{aligned}
$$

Denote the set of all such $m$-dimensional processes $f$ by $\mathcal{L}_{\phi}^{1,2}(m)$. As in the 1 dimensional case we obtain the isometries

$$
\mathbb{E}\left[\left(\int_{\mathbb{R}} f_{k} d B_{k}^{(H)}\right)^{2}\right]=\left\|f_{k}\right\|_{\mathcal{L}_{\phi_{k}}^{1,2}} ; \quad k=1,2, \ldots, m .
$$

This is intuitively clear, since we (by independence of $B_{1}^{(H)}, \ldots, B_{m}^{(H)}$ ) can treat the remaining stochastic variables $\omega_{1}, \ldots, \omega_{k-1}, \omega_{k+1}, \ldots, \omega_{m}$ as parameters and repeat the 1-dimensional approach in the $\omega_{k}$ variable. It is also easy to prove (2.6) rigorously by writing $f_{k}\left(t, \omega_{1}, \omega_{2}, \ldots, \omega_{m}\right)$ as a limit of sums of products of functions depending only on $\left(t, \omega_{k}\right)$ and only on $\left(\omega_{1}, \ldots, \omega_{k-1}, \omega_{k+1}, \ldots, \omega_{m}\right)$, respectively.

In view of this it is clear that if $f=\left(f_{1}, \ldots, f_{m}\right) \in \mathcal{L}_{\phi}^{1,2}(m)$, then the Wick-Itô integral (2.4) is well-defined as an element of $L^{2}(\mu)$ and by (2.6) we have

$$
\left\|\int_{\mathbb{R}} f d B^{(H)}\right\|_{L^{2}(\mu)} \leq \sum_{k=1}^{m}\left\|f_{k}\right\|_{\mathcal{L}_{\phi_{k}}^{1,2}}
$$

It is useful to have an explicit expression for the norm on the left hand side of (2.7). The following formula is our main result of this section:

Theorem 2.1 (Multi-dimensional fractional Wick-Itô Isometry I). Let $f, g \in$ $\mathcal{L}_{\phi}^{1,2}(m)$. Then

$$
\mathbb{E}\left[\left(\int_{\mathbb{R}} f d B^{(H)}\right) \cdot\left(\int_{\mathbb{R}} g d B^{(H)}\right)\right]=(f, g)_{\mathcal{L}_{\phi}^{1,2}(m)}
$$

where

$$
\begin{aligned}
& (f, g)_{\mathcal{L}_{\phi}^{1,2}(m)} \\
& =\mathbb{E}\left[\sum_{k=1}^{m} \int_{\mathbb{R}} \int_{\mathbb{R}} f_{k}(s) g_{k}(t) \phi_{k}(s, t) d s d t+\sum_{k, \ell=1}^{m}\left(\int_{\mathbb{R}} D_{\ell, t}^{\phi} f_{k}(t) d t\right) \cdot\left(\int_{\mathbb{R}} D_{k, t}^{\phi} g_{\ell}(t) d t\right)\right] .
\end{aligned}
$$

REMARK. Note the crossing of the indices $\ell, k$ of the derivatives and the components $f_{k}, g_{\ell}$ in the last terms of the right hand side of (2.9).

To prove Theorem 2.1 we proceed as in [6], but with the appropriate modifications:

In the 1-dimensional case, let $L_{\phi_{k}}^{2}$ be the set of deterministic functions $\alpha: \mathbb{R} \rightarrow \mathbb{R}$ such that

$$
(\alpha, \alpha)_{\phi_{k}}:=|\alpha|_{\phi_{k}}^{2}:=\int_{\mathbb{R}} \int_{\mathbb{R}} \alpha(s) \alpha(t) \phi_{k}(s, t) d s d t<\infty .
$$


If $\alpha \in L_{\phi_{k}}^{2}$ then clearly $\alpha \in \mathcal{L}_{\phi_{k}}^{1,2}$. Hence we can define the Wick (or Doleans-Dale) exponential

$$
\mathcal{E}(\alpha)=\exp ^{\diamond}\left(\int_{\mathbb{R}} \alpha(t) d B_{k}^{(H)}(t)\right)=\exp \left(\int_{\mathbb{R}} \alpha(t) d B_{k}^{(H)}(t)-\frac{1}{2}|\alpha|_{\phi_{k}}^{2}\right) .
$$

See e.g. [6, (3.1)] or [9, Example 3.10].

Similarly, in the multidimensional case we put $\phi=\left(\phi_{1}, \ldots, \phi_{m}\right)$ and we let $L_{\phi}^{2}$ be the set of all deterministic functions $\alpha=\left(\alpha_{1}, \ldots, \alpha_{m}\right): \mathbb{R} \rightarrow \mathbb{R}^{m}$ such that $\alpha_{k} \in L_{\phi_{k}}^{2}$ for $k=1, \ldots, m$. If $\alpha \in L_{\phi}^{2}$ we define the corresponding Wick exponential

$$
\begin{aligned}
\mathcal{E}(\alpha) & =\exp ^{\diamond}\left(\int_{\mathbb{R}} \alpha(t) d B^{(H)}(t)\right)=\exp ^{\diamond}\left(\sum_{k=1}^{m} \int_{\mathbb{R}} \alpha_{k}(t) d B_{k}^{(H)}(t)\right) \\
& =\exp \left(\sum_{k=1}^{m} \int_{\mathbb{R}} \alpha_{k}(t) d B_{k}^{(H)}(t)-\frac{1}{2}|\alpha|_{\phi}^{2}\right),
\end{aligned}
$$

where

$$
|\alpha|_{\phi}^{2}=\sum_{k=1}^{m} \int_{\mathbb{R}} \alpha_{k}(s) \alpha_{k}(t) \phi_{k}(s, t) d s d t=\sum_{k=1}^{m}|\alpha|_{\phi_{k}}^{2} .
$$

Let $\mathcal{E}$ be the linear span of all $\mathcal{E}(\alpha) ; \alpha \in L_{\phi}^{2}$. Then we have

Theorem 2.2. ([6, Theorem 3.1]) $\mathcal{E}$ is a dense subset of $L^{p}(\mathcal{F}, \mu)$, for all $p \geq 1$. and

TheOrem 2.3. ([6, Theorem 3.2]) Let $g_{i}=\left(g_{i 1}, \ldots, g_{i m}\right) \in L_{\phi}^{2}$ for $i=1,2, \ldots, n$ such that

$$
\left|g_{i k}-g_{j k}\right|_{\phi_{k}} \neq 0 \quad \text { if } \quad i \neq j, \quad k=1, \ldots, m .
$$

Then $\mathcal{E}\left(g_{1}\right), \ldots, \mathcal{E}\left(g_{n}\right)$ are linearly independent in $L^{2}(\mathcal{F}, \mu)$.

If $F \in L^{2}(\mathcal{F}, \mu)$ and $g_{k} \in L_{\phi_{k}}^{2}$ we put, as in [6],

$$
D_{k, \Phi\left(g_{k}\right)} F=\int_{\mathbb{R}} D_{k, t}^{\phi} F \cdot g_{k}(t) d t .
$$

We list some useful differentiation and Wick product rules. The proofs are similar to the 1-dimensional case and are omitted.

Lemma 2.4. Let $f=\left(f_{1}, \ldots, f_{m}\right) \in L_{\phi}^{2}, g=\left(g_{1}, \ldots, g_{m}\right) \in L_{\phi}^{2}$. Then

(i) $D_{k, \Phi\left(g_{k}\right)}\left(\sum_{i=1}^{m} \int_{\mathbb{R}} f_{i} d B_{i}^{(H)}\right)=\left(f_{k}, g_{k}\right)_{\phi_{k}}, \quad k=1, \ldots, m$, where

$$
\left(f_{k}, g_{k}\right)_{\phi_{k}}=\int_{\mathbb{R}} \int_{\mathbb{R}} f_{k}(s) g_{k}(t) \phi_{k}(s, t) d s d t ; \quad k=1, \ldots, m,
$$

(ii) $D_{k, s}^{\phi}\left(\sum_{i=1}^{m} \int_{\mathbb{R}} f_{i} d B_{i}^{(H)}\right)=\int_{\mathbb{R}} f_{k}(u) \phi_{k}(s, u) d u ; \quad k=1, \ldots, m$, 
(iii) $D_{k, \Phi\left(g_{k}\right)} \mathcal{E}(f)=\mathcal{E}(f) \cdot\left(f_{k}, g_{k}\right)_{\phi_{k}} ; \quad k=1, \ldots, m$,

(iv) $D_{k, s}^{\phi} \mathcal{E}(f)=\mathcal{E}(f) \cdot \int_{\mathbb{R}} f_{k}(u) \phi_{k}(s, u) d u ; \quad k=1, \ldots, m$,

(v) $\mathcal{E}(f) \diamond \mathcal{E}(g)=\mathcal{E}(f+g)$

(vi) $F \diamond \int_{\mathbb{R}} g_{k} d B_{k}^{(H)}=F \cdot \int_{\mathbb{R}} g_{k} d B_{k}^{(H)}-D_{k, \Phi\left(g_{k}\right)} F, \quad k=1, \ldots, m$,

(vii) $\mathbb{E}[\mathcal{E}(f) \cdot \mathcal{E}(g)]=\exp (f, g)_{\phi}$.

We now turn to the multi-dimensional case. We will prove

Lemma 2.5. Suppose $\alpha_{k} \in L_{\phi_{k}}^{2}, \beta_{\ell} \in L_{\phi_{\ell}}^{2}, D_{\ell, \Phi\left(\beta_{\ell}\right)} F \in L^{2}(\mu)$ and $D_{k, \Phi\left(\alpha_{k}\right)} G \in$ $L^{2}(\mu)$. Then

$$
\begin{array}{r}
\mathbb{E}\left[\left(F \diamond \int_{\mathbb{R}} \alpha_{k} d B_{k}^{(H)}\right) \cdot\left(G \diamond \int_{\mathbb{R}} \beta_{\ell} d B_{\ell}^{(H)}\right)\right] \\
=\mathbb{E}\left[\left(D_{\ell, \Phi\left(\beta_{\ell}\right)} F\right) \cdot\left(D_{k, \Phi\left(\alpha_{k}\right)} G\right)+\delta_{k \ell} F G\left(\alpha_{k}, \beta_{k}\right)_{\phi_{k}}\right],
\end{array}
$$

where

$$
\delta_{k \ell}= \begin{cases}1 & \text { if } k=\ell \\ 0 & \text { otherwise }\end{cases}
$$

Proof. We adapt the argument in [6] to the multi-dimensional case:

First note that by a density argument we may assume that

$$
F=\mathcal{E}(f)=\exp \left\{\int_{\mathbb{R}} f(t) d B^{(H)}(t)-\frac{1}{2}|f|_{\phi}^{2}\right\}
$$

and

$$
G=\mathcal{E}(g)=\exp \left\{\int_{\mathbb{R}} g(t) d B^{(H)}(t)-\frac{1}{2}|g|_{\phi}^{2}\right\},
$$

for some $f \in L_{\phi}^{2}, g \in L_{\phi}^{2}$.

Choose $\delta=\left(\delta_{1}, \ldots, \delta_{m}\right) \in \mathbb{R}^{m}, \gamma=\left(\gamma_{1}, \ldots, \gamma_{m}\right) \in \mathbb{R}^{m}$ and put $\delta \times f=$ $\left(\delta_{1} f_{1}, \ldots, \delta_{m} f_{m}\right)$ and $\gamma \times g=\left(\gamma_{1} g_{1}, \ldots, \gamma_{m} g_{m}\right)$. Then by Lemma 2.4

$$
\begin{aligned}
\mathbb{E} & {[(\mathcal{E}(f) \diamond \mathcal{E}(\delta \times \alpha)) \cdot(\mathcal{E}(g) \diamond \mathcal{E}(\gamma \times \beta))] } \\
& =\mathbb{E}[\mathcal{E}(f+\delta \times \alpha) \cdot \mathcal{E}(g+\gamma \times \beta)]=\exp (f+\delta \times \alpha, g+\gamma \times \beta)_{\phi} \\
& =\exp \left\{\sum_{i=1}^{m} \int_{\mathbb{R}} \int_{\mathbb{R}}\left(f_{i}+\delta_{i} \alpha_{i}\right)(s)\left(g_{i}+\gamma_{i} \beta_{i}\right)(t) \phi_{i}(s, t) d s d t\right\} .
\end{aligned}
$$

We now compute the double derivatives

$$
\frac{\partial^{2}}{\partial \delta_{k} \partial \gamma_{\ell}}
$$

of (2.18) and (2.19) at $\delta=\gamma=0$. We distinguish between two cases: 
Case 1. $k \neq \ell$

Then if we differentiate (2.18) we get

$$
\begin{aligned}
\frac{\partial^{2}}{\partial \delta_{k} \partial \gamma_{\ell}} \mathbb{E} & {[(\mathcal{E}(f) \diamond \mathcal{E}(\delta \times \alpha)) \cdot(\mathcal{E}(g) \diamond \mathcal{E}(\gamma \times \beta))]_{\delta=\gamma=0} } \\
= & \left.\frac{\partial}{\partial \gamma_{\ell}} \mathbb{E}\left[\left(\mathcal{E}(f) \diamond \mathcal{E}(\delta \times \alpha) \diamond \int_{\mathbb{R}} \alpha_{k} d B_{k}^{(H)}\right)\right) \cdot(\mathcal{E}(g) \diamond \mathcal{E}(\gamma \times \beta))\right]_{\delta=\gamma=0} \\
& =\mathbb{E}\left[\left(\mathcal{E}(f) \diamond \int_{\mathbb{R}} \alpha_{k} d B_{k}^{(H)}\right) \cdot\left(\mathcal{E}(g) \diamond \int_{\mathbb{R}} \beta_{\ell} d B_{\ell}^{(H)}\right)\right] .
\end{aligned}
$$

On the other hand, if we differentiate (2.19) we get

$$
\begin{aligned}
& \frac{\partial^{2}}{\partial \delta_{k} \partial \gamma_{\ell}}\left[\exp (f+\delta \times \alpha, g+\gamma \times \beta)_{\phi}\right]_{\delta=\gamma=0} \\
& =\frac{\partial}{\partial \gamma_{\ell}}\left[\exp (f+\delta \times \alpha, g+\gamma \times \beta)_{\phi} \cdot \int_{\mathbb{R}} \int_{\mathbb{R}} \alpha_{k}(s)\left(g_{k}+\gamma_{k} \beta_{k}\right)(t) \phi_{k}(s, t) d s d t\right]_{\delta=\gamma=0} \\
& =\exp (f, g)_{\phi} \cdot \int_{\mathbb{R}} \int_{\mathbb{R}} \alpha_{k}(s) g_{k}(t) \phi_{k}(s, t) d s d t \cdot \int_{\mathbb{R}} \int_{\mathbb{R}} \beta_{\ell}(s) f_{\ell}(t) \phi_{\ell}(s, t) d s d t \\
& =\exp (f, g)_{\phi} \cdot\left(\alpha_{k}, g_{k}\right)_{\phi_{k}} \cdot\left(\beta_{\ell}, f_{\ell}\right)_{\phi_{\ell}} \\
& =\mathbb{E}\left[\mathcal{E}(f) \cdot\left(\beta_{\ell}, f_{\ell}\right)_{\phi_{\ell}} \cdot \mathcal{E}(g) \cdot\left(\alpha_{k}, g_{k}\right)_{\phi_{k}}\right] \\
& =\mathbb{E}\left[D_{\ell, \Phi\left(\beta_{\ell}\right)} \mathcal{E}(f) \cdot D_{k, \Phi\left(\alpha_{k}\right)} \mathcal{E}(g)\right] .
\end{aligned}
$$

This proves (2.17) in this case.

Case 2. $k=\ell$

In this case, if we differentiate $(2.18)$ we get

$$
\begin{aligned}
\frac{\partial^{2}}{\partial \delta_{k} \partial \gamma_{k}} \mathbb{E} & {[(\mathcal{E}(f) \diamond \mathcal{E}(\delta \times \alpha)) \cdot(\mathcal{E}(g) \diamond \mathcal{E}(\gamma \times \beta))]_{\delta=\gamma=0} } \\
= & \frac{\partial}{\partial \gamma_{k}} \mathbb{E}\left[\left(\mathcal{E}(f) \diamond \mathcal{E}(\delta \times \alpha) \diamond \int_{\mathbb{R}} \alpha_{k} d B_{k}^{(H)}\right) \cdot(\mathcal{E}(g) \diamond \mathcal{E}(\gamma \times \beta))\right]_{\delta=\gamma=0} \\
= & \mathbb{E}\left[\left(\mathcal{E}(f) \diamond \int_{\mathbb{R}} \alpha_{k} d B_{k}^{(H)}\right) \cdot\left(\mathcal{E}(g) \diamond \int_{\mathbb{R}} \beta_{k} d B_{k}^{(H)}\right)\right]
\end{aligned}
$$

On the other hand, if we differentiate (2.19) we get

$$
\begin{aligned}
& \frac{\partial^{2}}{\partial \delta_{k} \partial \gamma_{k}}\left[\exp (f+\delta \times \alpha, g+\gamma \times \beta)_{\phi}\right]_{\delta=\gamma=0} \\
& =\frac{\partial}{\partial \gamma_{k}}\left[\exp (f+\delta \times \alpha, g+\gamma \times \beta)_{\phi} \cdot \int_{\mathbb{R}} \int_{\mathbb{R}} \alpha_{k}(s)\left(g_{k}+\gamma_{k} \beta_{k}\right)(t) \phi_{k}(s, t) d s d t\right]_{\delta=\gamma=0} \\
& =\exp (f, g)_{\phi} \cdot\left[\left(\alpha_{k}, g_{k}\right)_{\phi_{k}} \cdot\left(\beta_{k}, f_{k}\right)_{\phi_{k}}+\int_{\mathbb{R}} \int_{\mathbb{R}} \alpha_{k}(s) \beta_{k}(t) \phi_{k}(s, t) d s d t\right] \\
& =\mathbb{E}\left[D_{k, \Phi\left(\beta_{k}\right)} \mathcal{E}(f) \cdot D_{k, \Phi\left(\alpha_{k}\right)} \mathcal{E}(g)+\mathcal{E}(f) \mathcal{E}(g)\left(\alpha_{k}, \beta_{k}\right)_{\phi_{k}}\right] .
\end{aligned}
$$

This proves (2.17) also for Case 2 and the proof of Lemma 2.5 is complete.

We are now ready to prove Theorem 2.1: 
Proof. We may consider $\int_{\mathbb{R}} f_{k}(t) d B_{k}^{(H)}(t)$ as the limit of sums of the form

$$
\sum_{i=1}^{N} f_{k}\left(t_{i}\right) \diamond\left(B_{k}^{(H)}\left(t_{i+1}\right)-B_{k}^{(H)}\left(t_{i}\right)\right)
$$

when $\Delta t_{i}=t_{i+1}-t_{i} \rightarrow 0, t_{1}<t_{2}<\cdots<t_{N}, N=2,3, \ldots$ Hence $\mathbb{E}\left[\left(\int_{\mathbb{R}} f d B^{(H)}\right)^{2}\right]=\mathbb{E}\left[\left(\sum_{k=1}^{m} \int_{\mathbb{R}} f_{k} d B_{k}^{(H)}\right)^{2}\right]$ is the limit of sums of the form

$$
\sum_{i, j, k, \ell} \mathbb{E}\left[\left(f_{k}\left(t_{i}\right) \diamond\left(B_{k}^{(H)}\left(t_{i+1}\right)-B_{k}^{(H)}\left(t_{i}\right)\right)\right) \cdot\left(f_{\ell}\left(t_{j}\right) \diamond\left(B_{\ell}^{(H)}\left(t_{j+1}\right)-B_{\ell}^{(H)}\left(t_{j}\right)\right)\right]\right.
$$

which by Lemma 2.5 is equal to

$$
\sum_{i, j, k, \ell} \mathbb{E}\left[\left(\int_{t_{i}}^{t_{i+1}} D_{\ell, t}^{\phi} f_{k}\left(t_{i}\right) d t\right) \cdot\left(\int_{t_{j}}^{t_{j+1}} D_{k, t}^{\phi} f_{\ell}\left(t_{j}\right) d t\right)+\delta_{k \ell} \int_{t_{i}}^{t_{i+1}} \int_{t_{j}}^{t_{j+1}} f_{k}\left(t_{i}\right) f_{k}\left(t_{j}\right) \phi_{k}(s, t) d s d t\right] .
$$

When $\Delta t_{i} \rightarrow 0$ this converges to

$$
\mathbb{E}\left[\sum_{k, \ell=1}^{m}\left(\int_{\mathbb{R}} D_{\ell, t}^{\phi} f_{k}(t) d t\right) \cdot\left(\int_{\mathbb{R}} D_{k, t}^{\phi} f_{\ell}(t) d t\right)+\sum_{k=1}^{m} \int_{\mathbb{R}} \int_{\mathbb{R}} f_{k}(s) f_{k}(t) \phi_{k}(s, t) d s d t\right]
$$

This proves (2.9) when $f=g$. By polarization the proof of Theorem 2.1 is complete. 口

Using Theorem 2.1 we can now proceed as in the 1-dimensional case ([6, Theorem 4.3]), with appropriate modifications, and obtain a fractional multi-dimensional Itô formula. We omit the proof.

Theorem 2.6 (The fractional multi-dimensional Itô formula). Let $X(t)=$ $\left(X_{1}(t), \ldots, X_{n}(t)\right)$, with

$$
\begin{aligned}
& d X_{i}(t)=\sum_{j=1}^{m} \sigma_{i j}(t, \omega) d B_{j}^{(H)}(t) \\
& \quad \text { where } \sigma_{i}=\left(\sigma_{i 1}, \ldots, \sigma_{i m}\right) \in \mathcal{L}_{\phi}^{1,2}(m) ; \quad 1 \leq i \leq n .
\end{aligned}
$$

Suppose that for all $j=1, \ldots, m$ there exists $\theta_{j}>1-H_{j}$ such that

$$
\sup _{i} \mathbb{E}\left[\left(\sigma_{i j}(u)-\sigma_{i j}(v)\right)^{2}\right] \leq C|u-v|^{\theta_{j}} \quad \text { if }|u-v|<\delta
$$

where $\delta>0$ is a constant. Moreover, suppose that

$$
\lim _{\substack{0 \leq u, v \leq t \\|u-v| \rightarrow 0}}\left\{\sup _{i, j, k} \mathbb{E}\left[\left(D_{k, u}^{\phi}\left\{\sigma_{i j}(u)-\sigma_{i j}(v)\right\}\right)^{2}\right]=0 .\right.
$$

Let $f \in C^{1,2}\left(\mathbb{R} \times \mathbb{R}^{n}\right)$ with bounded second order derivatives with respect to $x$. Then, 
for $t>0$,

$$
\begin{aligned}
f(t, X(t)) & =f(0, X(0))+\int_{0}^{t} \frac{\partial f}{\partial s}(s, X(s)) d s+\int_{0}^{t} \sum_{i=1}^{n} \frac{\partial f}{\partial x_{i}}(s, X(s)) d X_{i}(s) \\
& +\int_{0}^{t}\left\{\sum_{i, j=1}^{m} \frac{\partial^{2} f}{\partial x_{i} \partial x_{j}}(s, X(s)) \sum_{k=1}^{m} \sigma_{i k}(s) D_{k, s}^{\phi}\left(X_{j}(s)\right)\right\} d s \\
& =f(0, X(0))+\int_{0}^{t} \frac{\partial f}{\partial s}(s, X(s)) d s+\sum_{j=1}^{m} \int_{0}^{t}\left[\sum_{i=1}^{n} \frac{\partial f}{\partial x_{i}}(s, X(s)) \sigma_{i j}(s, \omega)\right] d B_{j}^{(H)}(s) \\
& +\int_{0}^{t} \operatorname{Tr}\left[\Lambda^{T}(s) f_{x x}(s, X(s))\right] d s .
\end{aligned}
$$

Here $\Lambda=\left[\Lambda_{i j}\right] \in \mathbb{R}^{n \times m}$ with

$$
\begin{gathered}
\Lambda_{i j}(s)=\sum_{k=1}^{m} \sigma_{i k} D_{k, s}^{\phi}\left(X_{j}(s)\right) ; \quad 1 \leq i \leq n, \quad 1 \leq j \leq m, \\
f_{x x}=\left[\frac{\partial^{2} f}{\partial x_{i} \partial x_{j}}\right]_{1 \leq i, j \leq n} \in \mathbb{R}^{n \times n}
\end{gathered}
$$

and $(\cdot)^{T}$ denotes matrix transposed, $\operatorname{Tr}[\cdot]$ denotes matrix trace.

If we combine Theorem 2.6 with Theorem 2.1 we get the following result, which also may be regarded as a fractional Itô isometry:

Theorem 2.7 (Fractional Itô isometry II). Suppose $f=\left(f_{1}, \ldots, f_{m}\right) \in \mathcal{L}_{\phi}^{1,2}(m)$. Then, for $T>0$,

$$
\begin{aligned}
\mathbb{E} & {\left[\left(\int_{0}^{T} D_{\ell, t}^{\phi} f_{k}(t) d t\right) \cdot\left(\int_{0}^{T} D_{k, t}^{\phi} f_{\ell}(t) d t\right)\right] } \\
& =\mathbb{E}\left[\int_{0}^{T}\left\{f_{k}(t) \int_{0}^{t} D_{k, t}^{\phi} f_{\ell}(s) d B_{\ell}^{(H)}(s)+f_{\ell}(t) \int_{0}^{T} D_{\ell, t}^{\phi} f_{k}(s) d B_{k}^{(H)}(s)\right\} d t\right]
\end{aligned}
$$

Proof. By the Itô formula (Theorem 2.6) we have

$$
\begin{aligned}
\mathbb{E} & {\left[\left(\int_{0}^{T} f_{k} d B_{k}^{(H)}\right) \cdot\left(\int_{0}^{T} f_{\ell} d B_{\ell}^{(H)}\right)\right] } \\
& =\mathbb{E}\left[\int_{0}^{T}\left\{f_{k}(t) D_{k, t}^{\phi}\left(\int_{0}^{t} f_{\ell}(s) d B_{\ell}^{(H)}(s)\right)+f_{k}(t) D_{\ell, t}^{\phi}\left(\int_{0}^{t} f_{k}(s) d B_{k}^{(H)}(s)\right)\right\} d t\right] \\
& =\mathbb{E}\left[\int_{0}^{T}\left\{f_{k}(t) \int_{0}^{t} D_{k, t}^{\phi} f_{\ell}(s) d B_{\ell}^{(H)}(s)+f_{\ell}(t) \int_{0}^{t} D_{\ell, t}^{\phi} f_{k}(s) d B_{k}^{(H)}(s)\right\} d t\right] \\
& +\delta_{k \ell} \mathbb{E}\left[\int_{0}^{T} \int_{0}^{t}\left\{f_{k}(t) f_{k}(s)+f_{\ell}(t) f_{k}(s)\right\} \phi_{k}(s, t) d s d t\right]
\end{aligned}
$$

where we have used that, for $u>0$,

$$
D_{k, t}^{\phi}\left(\int_{0}^{u} f_{\ell}(s) d B_{\ell}^{(H)}(s)\right)=\int_{0}^{u} D_{k, t}^{\phi} f_{\ell}(s) d B_{\ell}^{(H)}(s)+\delta_{k \ell} \int_{0}^{u} f_{k}(s) \phi_{k}(t, s) d s .
$$


(See [6, Theorem 4.2].)

On the other hand, the Itô isometry (Theorem 2.1) gives that

$$
\begin{aligned}
& \mathbb{E}\left[\left(\int_{0}^{T} f_{k} d B_{k}^{(H)}\right) \cdot\left(\int_{0}^{T} f_{\ell} d B_{\ell}^{(H)}\right)\right] \\
& \quad=\mathbb{E}\left[\left(\int_{0}^{T} D_{\ell, t}^{\phi} f_{k}(t) d t\right) \cdot\left(\int_{0}^{T} D_{k, t}^{\phi} f_{\ell}(t) d t\right)+\delta_{k \ell}\left|f_{k}\right|_{\phi_{k}}^{2}\right] .
\end{aligned}
$$

Comparing (2.33) and (2.35) we get Theorem 2.7.

We end this section by proving a fractional integration by parts formula. First we recall

TheOREm 2.8 (Fractional Girsanov formula). Suppose $\gamma=\left(\gamma_{1}, \ldots, \gamma_{m}\right) \in$ $\left(L^{2}(\mathbb{R})\right)^{m}$ and $\hat{\gamma}=\left(\hat{\gamma}_{1}, \ldots, \hat{\gamma}_{m}\right) \in L_{\phi}^{2}$ are related by

$$
\gamma_{k}(t)=\int_{\mathbb{R}} \hat{\gamma}_{k}(s) \phi_{k}(s, t) d s ; \quad t \in \mathbb{R}, \quad k=1, \ldots, m .
$$

Let $G \in L^{2}(\mu)$. Then

$$
\mathbb{E}[G(\omega+\gamma)]=\mathbb{E}\left[G(\omega) \exp ^{\diamond}(\langle\omega, \hat{\gamma}\rangle)\right]=\mathbb{E}\left[G(\omega) \mathcal{E}\left(\int_{\mathbb{R}} \hat{\gamma} d B^{(H)}\right)\right] .
$$

For a proof in the 1-dimensional case see e.g. [9, Theorem 3.16]. The proof in the multi-dimensional case is similar.

If $F \in L^{2}(\mu)$ and $\gamma=\left(\gamma_{1}, \ldots, \gamma_{m}\right) \in\left(L^{2}(\mathbb{R})\right)^{m}$ the directional derivative of $F$ in the direction $\gamma$ is defined by

$$
D_{\gamma} F(\omega)=\lim _{\varepsilon \rightarrow 0} \frac{F(\omega+\varepsilon \gamma)-F(\omega)}{\varepsilon},
$$

provided the limit exists in $L^{2}(\mu)$. We say that $F$ is differentiable if there exists a process $D_{t} F(\omega)=\left(D_{1, t} F(\omega), \ldots, D_{m, t} F(\omega)\right)$ such that $D_{k, t} F(\omega) \in L^{2}(d \mu \otimes d t)$ for all $k=1, \ldots, m$ and

$$
D_{\gamma} F(\omega)=\int_{\mathbb{R}} D_{t} F(\omega) \cdot \gamma(t) d t \quad \text { for all } \gamma \in\left(L^{2}(\mathbb{R})\right)^{m} .
$$

TheOREM 2.9 (Fractional integration by parts I). Let $F, G \in L^{2}(\mu), \gamma \in$ $\left(L^{2}(\mathbb{R})\right)^{m}$ and assume that the directional derivatives $D_{\gamma} F, D_{\gamma} G$ exist. Then

$$
\mathbb{E}\left[D_{\gamma} F \cdot G\right]=\mathbb{E}\left[F \cdot G \cdot \int_{\mathbb{R}} \hat{\gamma} d B^{(H)}\right]-\mathbb{E}\left[F \cdot D_{\gamma} G\right] .
$$

Proof. By Theorem 2.8 we have, for all $\varepsilon>0$,

$$
\mathbb{E}[F(\omega+\varepsilon \gamma) G(\omega)]=\mathbb{E}\left[F(\omega) G(\omega-\varepsilon \gamma) \exp ^{\diamond}(\varepsilon\langle\omega, \hat{\gamma}\rangle)\right] .
$$


Hence

$$
\begin{aligned}
\mathbb{E}\left[D_{\gamma} F \cdot G\right] & =\mathbb{E}\left[\lim _{\varepsilon \rightarrow 0} \frac{1}{\varepsilon}\{F(\omega+\varepsilon \gamma)-F(\omega)\} G(\omega)\right] \\
& =\mathbb{E}\left[\lim _{\varepsilon \rightarrow 0} \frac{1}{\varepsilon}\left\{F(\omega)\left[G(\omega-\varepsilon \gamma) \exp ^{\diamond}(\varepsilon\langle\omega, \hat{\gamma}\rangle)-G(\omega)\right]\right\}\right] \\
& =\mathbb{E}\left[F(\omega) \frac{d}{d \varepsilon}\left\{G(\omega-\varepsilon \gamma) \exp \left(\varepsilon \int_{\mathbb{R}} \hat{\gamma} d B^{(H)}-\frac{1}{2} \varepsilon^{2}|\hat{\gamma}|_{\phi}^{2}\right)\right\}_{\varepsilon=0}\right] \\
& =\mathbb{E}\left[F(\omega) G(\omega) \int_{\mathbb{R}} \hat{\gamma} d B^{(H)}\right]-\mathbb{E}\left[F(\omega) D_{\gamma} G(\omega)\right]
\end{aligned}
$$

$\square$

We now apply the above to the fractional gradient

$$
D_{t}^{\phi} F=\int_{\mathbb{R}} D_{s} F \cdot \phi(s, t) d s=\sum_{k=1}^{m} \int_{\mathbb{R}} D_{k, s} F \cdot \phi_{k}(s, t) d s=D_{\phi} F(\omega)
$$

Theorem 2.10 (Fractional integration by parts II). Suppose F, $G \in L^{2}(\mu)$ are differentiable, with fractional gradients $D_{t}^{\phi} F, D_{t}^{\phi} G$. Then for each $t \in \mathbb{R}, k \in\{1, \ldots, m\}$ we have

$$
\mathbb{E}\left[D_{k, t}^{\phi} F \cdot G\right]=\mathbb{E}\left[F \cdot G \cdot B_{k}^{(H)}(t)\right]-\mathbb{E}\left[F \cdot D_{k, t}^{\phi} G\right]
$$

Proof. Choose a sequence $\hat{\gamma}_{k}^{(j)} \in L_{\phi_{k}}^{2} ; j=1,2, \ldots$, such that $\lim _{j \rightarrow \infty} \hat{\gamma}_{k}^{(j)}=\delta_{t}(\cdot)($ the point mass at $t$ ), in the sense that if we define

$$
\phi_{k}^{(j)}(s)=\int_{\mathbb{R}} \hat{\gamma}_{k}^{(j)} \phi_{k}(s, r) d r
$$

then $\phi_{k}^{(j)}(\cdot) \rightarrow \phi_{k}(\cdot, t)$ in $L^{2}(\mathbb{R})$. Then by Theorem 2.9

$$
\begin{aligned}
\mathbb{E}\left[D_{k, t}^{\phi} F \cdot G\right] & =\mathbb{E}\left[\lim _{j \rightarrow \infty} D_{\phi_{k}^{(j)}} F \cdot G\right]=\lim _{j \rightarrow \infty} \mathbb{E}\left[D_{\phi_{k}^{(j)}} F \cdot G\right] \\
& =\lim _{j \rightarrow \infty} \mathbb{E}\left[F \cdot G \cdot \int_{\mathbb{R}} \hat{\gamma}^{(j)} d B^{(H)}\right]-\mathbb{E}\left[F \cdot D_{\phi_{k}^{(j)}} G\right] \\
& =\mathbb{E}\left[F \cdot G \cdot B_{k}^{(H)}(t)\right]-\mathbb{E}\left[F \cdot D_{k, t} G\right] .
\end{aligned}
$$

$\square$

3. Application to minimal variance hedging. Consider the multidimensional version of the fractional mathematical market model introduced by [9] and by [7], consisting of $n+1$ independent fractional Brownian motions $B_{1}^{(H)}(t), \ldots, B_{m}^{(H)}(t)$ with Hurst coefficients $H_{1}, \ldots, H_{m}$ respectively $\left(\frac{1}{2}<H_{i}<1\right)$, as follows:

$$
\begin{aligned}
\text { (bond price) } & d S_{0}(t)=r(t, \omega) d t ; \quad S_{0}(0)=s_{0}, \quad 0 \leq t \leq T \\
\text { (stock prices) } & d S_{i}(t)=\mu_{i}(t, \omega) d t+\sum_{j=1}^{m} \sigma_{i j}(t, \omega) d B_{j}^{(H)}(t) ; \quad S_{i}(0)=s_{i}, \\
i & =1, \ldots, n, \quad 0 \leq t \leq T .
\end{aligned}
$$


Here $r(t, \omega), \mu_{i}(t, \omega)$ and $\sigma_{i j}(t, w)$ are $\mathcal{F}_{t}^{(H)}$-adapted processes satisfying reasonable growth conditions. We refer to [7], [9], [14] and [21] for a general discussion of such markets.

We say that $g=\left(g_{1}, \ldots, g_{m}\right)$ is an admissible portfolio if $g(t)$ is $\mathcal{F}_{t}^{(H)}$-adapted, $g \sigma \in \mathcal{L}_{\phi}^{1,2}(m)$ and $\mathbb{E}\left[\int_{0}^{T} \sum_{i=1}^{n}\left|g_{i}(t) \mu_{i}(t)\right| d t\right]<\infty$. Here we denote by $\sigma$ the volatility matrix $[\sigma]_{i, j}(\cdot)=\sigma_{i j}(\cdot)$. Suppose we are only allowed to trade in some, say $k$, of the securities $S_{0}, \ldots, S_{n}$. Let $\mathcal{K}$ be the set of $i \in\{1, \ldots, n\}$ such that trading in $S_{i}$ is allowed. Then, according to our model, the wealth hedged by an initial value $z \in \mathbb{R}$ and an admissible portfolio $g(t)=\left(g_{i}(t, \omega)\right)_{i \in \mathcal{K}} \in \mathbb{R}^{k}$ up to time $t$ is

$$
V(t)=V_{z}^{g}(t)=z+\sum_{i \in \mathcal{K}} \int_{0}^{t} g_{i}(u) d S_{i}(u) ; \quad 0 \leq t \leq T .
$$

Now let $F(\omega)$ be a $T$-claim, i.e. an $\mathcal{F}_{T}^{(H)}$-measurable random variable in $L^{2}(\mu)$.

The minimal variance hedging problem is to find a $z^{*} \in \mathbb{R}$ and an admissible portfolio $g^{*}$ such that

$$
\mathbb{E}\left[\left(F-V_{z^{*}}^{g^{*}}(T)\right)^{2}\right]=\inf _{z, g} \mathbb{E}\left[\left(F-V_{z}^{g}(T)\right)^{2}\right] .
$$

This is a difficult problem even in the classical Brownian motion setting. See e.g. [8], [17] and the references therein. For a recent general martingale approach see [5]. For fractional Brownian motion markets a special case is solved in [1] by using optimal control theory.

Here we will discuss the two-dimensional case only, and we will simply assume that

$$
d S_{0}(t)=0, \quad d S_{1}(t)=d B_{1}^{(H)}(t) \quad \text { and } \quad d S_{2}(t)=d B_{2}^{(H)}(t)
$$

Assume that only trading in $S_{0}$ and $S_{1}$ is allowed. Then the problem is to minimize

$$
J\left(z, g_{1}\right)=\mathbb{E}\left[\left(F-\left(z+\int_{0}^{T} g_{1} d S_{1}\right)\right)^{2}\right]
$$

over all $z \in \mathbb{R}$ and all admissible portfolios $g_{1}$.

By the fractional Clark-Haussmann-Ocone formula ([9, Theorem 4.15]) we can write

$$
F(\omega)=\mathbb{E}[F]+\int_{0}^{T} f_{1}(t) d B_{1}^{(H)}(t)+\int_{0}^{T} f_{2}(t) d B_{2}^{(H)}(t)
$$

where

$$
f_{i}(t)=\widetilde{\mathbb{E}}\left[D_{i, t} F \mid \mathcal{F}_{t}^{(H)}\right] ; \quad i=1,2 .
$$

Substituting this into (3.5) we get, by (1.8),

$$
\begin{aligned}
J\left(z, g_{1}\right) & =\mathbb{E}\left[\left(\mathbb{E}[F]-z+\int_{0}^{T}\left(f_{1}-g_{1}\right) d B_{1}^{(H)}+\int_{0}^{T} f_{2} d B_{2}^{(H)}\right)^{2}\right] \\
& =(\mathbb{E}[F]-z)^{2}+\mathbb{E}\left[\left(\int_{0}^{T}\left(f_{1}-g_{1}\right) d B_{1}^{(H)}+\int_{0}^{T} f_{2} d B_{2}^{(H)}\right)^{2}\right] .
\end{aligned}
$$


Hence it is optimal to choose $z=z^{*}:=\mathbb{E}[F]$. The remaining problem is therefore to minimize

$$
J_{0}\left(g_{1}\right)=\mathbb{E}\left[\left(\int_{0}^{T}\left(f_{1}-g_{1}\right) d B_{1}^{(H)}+\int_{0}^{T} f_{2} d B_{2}^{(H)}\right)^{2}\right] .
$$

From now on we assume that $f_{1} \in \mathcal{L}_{\phi_{i}}^{1,2}$ for $i=1,2$. By a Hilbert space argument on $L^{2}(\mu)$ we see that $g_{1}^{*}$ minimizes (3.8) if and only if

$$
\begin{gathered}
\mathbb{E}\left[\left(\int_{0}^{T}\left(f_{1}-g_{1}\right) d B_{1}^{(H)}+\int_{0}^{T} f_{2} d B_{2}^{(H)}\right) \cdot\left(\int_{0}^{T} \gamma d B_{1}^{(H)}\right)\right]=0 \\
\text { for all adapted } \gamma \in \mathcal{L}_{\phi_{1}}^{1,2} .
\end{gathered}
$$

By Theorem 2.1 (3.9) is equivalent to

$$
\begin{gathered}
\mathbb{E}\left[\int_{0}^{T} \int_{0}^{T}\left(f_{1}(t)-g_{1}(t)\right) \gamma(s) \phi_{1}(s, t) d s d t+\left(\int_{0}^{T} D_{1, t}^{\phi}\left(f_{1}(t)-g_{1}(t)\right) d t\right)\left(\int_{0}^{T} D_{1, t}^{\phi} \gamma(t) d t\right)\right. \\
\left.\quad+\left(\int_{0}^{T} D_{1, t}^{\phi} f_{2}(t) d t\right) \cdot\left(\int_{0}^{T} D_{2, t}^{\phi} \gamma(t) d t\right)\right] \\
\quad=0 \quad \text { for all adapted } \gamma \in \mathcal{L}_{\phi}^{1,2} .
\end{gathered}
$$

From this we immediately deduce

Proposition 3.1. The portfolio

$$
g_{1}(t)=g_{1}^{*}(t):=f_{1}(t)
$$

minimizes (3.8) if and only if

$$
\int_{0}^{T} D_{1, t}^{\phi} f_{2}(t) d t=0 \quad \text { a.s. }
$$

This result is surprising in view of the corresponding situation for classical Brownian motion, when it is always optimal to choose $g_{1}(t)=g_{1}^{*}(t)=f_{1}(t)$.

We also get

Proposition 3.2. Suppose $g_{1}^{*}(t)$ minimizes (3.8). Then

$$
\mathbb{E}\left[\int_{0}^{T}\left(f_{1}(t)-g_{1}^{*}(t)\right) d t\right]=0 .
$$

Proof. This follows by choosing $\gamma(t)$ deterministic in (3.10). 
Now assume that $D_{1, t}^{\phi}\left(f_{1}(t)\right)$ and $D_{1, t}^{\phi}\left(g_{1}(t)\right)$ are differentiable with respect to $D_{1, s}^{\phi}$ and that $D_{1, t}^{\phi} f_{2}(t)$ is differentiable with respect to $D_{2, s}^{\phi}$ for all $s \in[0, T]$. Then we can use integration by parts (Theorem 2.10) to rewrite equation (3.10) as follows:

$$
\begin{aligned}
& \mathbb{E}\left[\int _ { 0 } ^ { T } \int _ { 0 } ^ { T } \left\{\left(f_{1}(t)-g_{1}(t)\right) \gamma(s) \phi_{1}(s, t)+D_{1, t}^{\phi}\left(f_{1}(t)-g_{1}(t)\right) \cdot D_{1, s}^{\phi} \gamma(s)\right.\right. \\
& \left.\left.\quad+D_{1, t}^{\phi} f_{2}(t) \cdot D_{2, s}^{\phi} \gamma(s)\right\} d s d t\right] \\
& =\int_{0}^{T} \int_{0}^{T} \mathbb{E}\left[\left(f_{1}(t)-g_{1}(t)\right) \phi_{1}(s, t) \gamma(s)+D_{1, t}^{\phi}\left(f_{1}(t)-g_{1}(t)\right) \gamma(s) B_{1}^{(H)}(s)\right. \\
& \quad-D_{1, s}^{\phi} D_{1, t}^{\phi}\left(f_{1}(t)-g_{1}(t)\right) \gamma(s)+D_{1, t}^{\phi} f_{2}(t) \gamma(s) B_{2}^{(H)}(s) \\
& \left.\quad-D_{2, s}^{\phi} D_{1, t}^{\phi} f_{2}(t) \gamma(s)\right] d s d t \\
& =\mathbb{E}\left[\int_{0}^{T} K(s) \gamma(s) d s\right]=0,
\end{aligned}
$$

where

$$
K(s)=\int_{0}^{T} G(s, t) d t
$$

with

$$
\begin{aligned}
G(s, t) & =\left(f_{1}(t)-g_{1}(t)\right) \phi_{1}(s, t)+D_{1, t}^{\phi}\left(f_{1}(t)-g_{1}(t)\right) B_{1}^{(H)}(s) \\
& -D_{1, s}^{\phi} D_{1, t}^{\phi}\left(f_{1}(t)-g_{1}(t)\right)+D_{1, t}^{\phi} f_{2}(t) B_{2}^{(H)}(s)-D_{2, s}^{\phi} D_{1, t}^{\phi} f_{2}(t) .
\end{aligned}
$$

Since $\gamma(s)$ is $\mathcal{F}_{s}^{(H)}$-measurable we get from (3.13) that

$$
\begin{aligned}
0 & =\int_{0}^{T} \mathbb{E}[K(s) \gamma(s)] d s=\int_{0}^{T} \mathbb{E}\left[\mathbb{E}\left[K(s) \gamma(s) \mid \mathcal{F}_{s}^{(H)}\right]\right] d s \\
& =\int_{0}^{T} \mathbb{E}\left[\gamma(s) \mathbb{E}\left[K(s) \mid \mathcal{F}_{s}^{(H)}\right]\right] d s=\mathbb{E}\left[\int_{0}^{T} \mathbb{E}\left[K(s) \mid \mathcal{F}_{s}^{(H)}\right] \gamma(s) d s\right] .
\end{aligned}
$$

Since this holds for all adapted $\gamma \in \mathcal{L}_{\phi}^{1,2}$ we conclude that

$$
\mathbb{E}\left[K(s) \mid \mathcal{F}_{s}^{(H)}\right]=0 \quad \text { for a.a. }(s, \omega) .
$$

or, using (3.14),

$$
\begin{aligned}
& \int_{0}^{T}\left\{\mathbb{E}_{s}\left[f_{1}(t)-g_{1}(t)\right] \phi_{1}(s, t)+\mathbb{E}_{s}\left[D_{1, t}^{\phi}\left(f_{1}\left(t-g_{1}(t)\right)\right] B_{1}^{(H)}(s)\right.\right. \\
& \left.-\mathbb{E}_{s}\left[D_{1, s}^{\phi} D_{1, t}^{\phi}\left(f_{1}(t)-g_{1}(t)\right)\right]+\mathbb{E}_{s}\left[D_{1, t}^{\phi} f_{2}(t)\right] B_{2}^{(H)}(s)-\mathbb{E}_{s}\left[D_{2, s}^{\phi} D_{1, t}^{\phi} f_{2}(t)\right]\right\} d t=0,
\end{aligned}
$$

where we have used the shorthand notation

$$
\mathbb{E}_{s}[\cdot]=\mathbb{E}\left[\cdot \mid \mathcal{F}_{s}^{(H)}\right] .
$$


We have proved:

THEOREM 3.3. Suppose the claim $F$ represented by (3.6) is such that $D_{1, s}^{\phi} D_{1, t}^{\phi} f_{1}(t)$ and $D_{2, s}^{\phi} D_{1, t}^{\phi} f_{2}(t)$ exist for all $s, t \in[0, T]$. Suppose $\hat{g}_{1}(t)$ is an adapted process in $\mathcal{L}_{\phi}^{1,2}$ such that $D_{1, t}^{\phi} \hat{g}_{1}(t)$ and $D_{1, s}^{\phi} D_{1, t}^{\phi} \hat{g}_{1}(t)$ exist for all $s, t \in[0, T]$. Then the following are equivalent:

(i) $\hat{g}_{1}(t)$ is a minimal variance hedging portfolio for $F$, i.e. $\hat{g}_{1}(t)$ minimizes (3.8) over all adapted $g_{1}(t) \in \mathcal{L}_{\phi}^{1,2}$

(ii) $g_{1}(t)=\hat{g}_{1}(t)$ satisfies equation (3.18).

Note that the same method also applies if we assume a fractional exponential dynamics for the asset prices, which represents a more realistic financial model. To illustrate this result we consider the following special case:

EXAMPLE 3.4. Suppose $f_{1}(t)=0$ and

$$
D_{1, t}^{\phi} f_{2}(t)=h(t), \quad \text { a deterministic function . }
$$

We seek a minimal variance hedging portfolio $g_{1}^{*}(t)$ for the claim

$$
F(\omega)=\int_{0}^{T} f_{2}(t) d B_{2}^{(H)}(t) .
$$

In this case (3.18) gets the form

$$
\begin{gathered}
\int_{0}^{T}\left\{-\mathbb{E}_{s}\left[g_{1}(t)\right] \phi_{1}(s, t)-\mathbb{E}_{s}\left[D_{1, t}^{\phi} g_{1}(t)\right] B_{1}^{(H)}(s)+\mathbb{E}_{s}\left[D_{1, s}^{\phi} D_{1, t}^{\phi} g_{1}(t)\right]\right. \\
\left.+h(t) B_{2}^{(H)}(s)\right\} d t=0 \quad \text { for a.a. }(s, \omega) .
\end{gathered}
$$

Let us try to choose $g_{1}(t)$ such that

$$
D_{1, t}^{\phi} g_{1}(t)=0
$$

Then (3.19) reduces to

$$
\int_{0}^{T} \mathbb{E}_{s}\left[g_{1}(t)\right] \phi_{1}(s, t) d t=B_{2}^{(H)}(s) \int_{0}^{T} h(t) d t
$$

or, since $g_{1}$ is adapted,

$$
\int_{0}^{s} g_{1}(t) \phi_{1}(s, t) d t+\int_{s}^{T} \mathbb{E}_{s}\left[g_{1}(t)\right] \phi_{1}(s, t) d t=B_{2}^{(H)}(s) \int_{0}^{T} h(t) d t, \quad s \in[0, T] .
$$

In particular, if we choose $s=T$ we get the equation

$$
\int_{0}^{T} g_{1}(t) \phi_{1}(T, t) d t=B_{2}^{(H)}(T) \int_{0}^{T} h(t) d t,
$$

which clearly has no adapted solution $g_{1}(t)$. (However, it obviously has a non-adapted solution.) Therefore an optimal portfolio $g_{1}(t)=g_{1}^{*}(t)$ for the claim (3.20), if it exists, cannot satisfy (3.22). 


\section{REFERENCES}

[1] F. Biagini, Y. Hu, B. Øksendal And A. Sulem, A stochastic maximum principle for processes driven by fractional Brownian motion, Stoch. Proc. and their Appl., 100 (2002), pp. 233253.

[2] F.Biagini, B.Øksendal, A.Sulem, N.Wallner, An introduction to White noise theory and Malliavin calculus for fractional Brownian motion Proceedings of the Royal Society of London (to appear).

[3] D. Brody, J. Syroka And M. Zervos, Pricing weather derivative options, Quantitative Finance, 2 (2002), pp. 189-198.

[4] A. Dasgupta, Fractional Brownian Motion: Its properties and applications to stochastic integration, Ph.D. Thesis, Dept. of Statistics, University of North Caroline at Chapel Hill 1997.

[5] G. Di Nunno, Stochastic integral representations, stochastic derivatives and minimal variance hedging, Stochastics and Stochastics Reports, 73 (2002), pp. 181-198.

[6] T. E. Duncan, Y. Hu And B. Pasik-Duncan, Stochastic calculus for fractional Brownian motion, I. Theory. SIAM J. Control Optim., 38 (2000), pp. 582-612.

[7] R.J. Elliott AND J. VAN DER HoEk, A general fractional white noise theory and applications to finance, Math. Finance, 13 (2003), pp. 301-330.

[8] H. Föllmer AND M. SchweIzer, Hedging of contingent claims under incomplete information, In M.H.A. Davis and R. Elliott (eds): Applied Stochastic Analysis, Gordon and Breach 1991, pp. 389-414.

[9] Y. Hu AND B. ØKSEndal, Fractional white noise calculus and applications to finance, Infinite dimensional analysis, quantum probability and related topics, 6:1 (2003), pp. 1-32.

[10] B. Mandelbrot, Fractals and Scaling in Finance: Discontinuity, Concentration, Risk, Springer-Verlag, 1997.

[11] B.B. Mandelbrot and J.W. van Ness, Fractional Brownian motions, fractional noises and applications, SIAM Rev., 10 (1968), pp. 422-437.

[12] R. NoRvaIS̆A, Modelling of stock price changes. A real analysis approach, Finance and Stochastics, 4 (2000), pp. 34-369.

[13] I. Norros, E. Valkeila and J. Virtamo, An elementary approach to a Girsanov formula and other analytic results on fractional Brownian motions, Bernoulli, 5 (1999), pp. 571-587.

[14] B. ØKsendal, Fractional Brownian motion in finance, Preprint, Department of Mathematics, University of Oslo 28/2003.

[15] L.C.G. Rogers, Arbitrage with fractional Brownian motion, Math. Finance, 7 (1997), pp. 95-105.

[16] D.M. SAlopeK, Tolerance to arbitrage, Stoch. Proc. and their Applications, 76 (1998), pp. $217-230$.

[17] M. SchweIzer, A guided tour through quadratic hedging approaches, In: Jouini E., Cvitanic J., Musiela M.(eds) Option pricing, interest rates and risk management.Cambridge, UK:Cambridge University Press 2001a, pp. 538-574.

[18] A. Shiryaev, Essentials of Stochastic Finance, World Scientific, 1999.

[19] A. ShiRyaev, On arbitrage and replication for fractal models, In A. Shiryaev and A. Sulem (eds.): Workshop on Mathematical Finance, INRIA, Paris, 1998.

[20] I. Simonsen, Anti-correlations in the Nordic electricity spot market, Manuscript, NORDITA Copenhagen, May 2001.

[21] N. Wallner, Fractional Brownian Motion and Applications to Finance, Thesis, PhilippsUniversität Marburg, March 2001. 